\title{
8
}

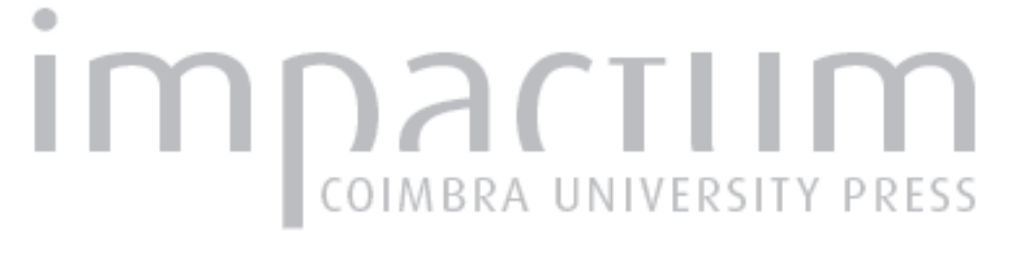

\section{Lingua e cultura italiana in Portogallo}

\section{Autor(es): [s.n.]}

Publicado por: Imprensa da Universidade de Coimbra

URL persistente: URI:http://hdl.handle.net/10316.2/42513

DOI: $\quad$ DOI:https://doi.org/10.14195/0870-8584_5_1

Accessed : $\quad$ 26-Apr-2023 03:09:02

A navegação consulta e descarregamento dos títulos inseridos nas Bibliotecas Digitais UC Digitalis, UC Pombalina e UC Impactum, pressupõem a aceitação plena e sem reservas dos Termos e Condições de Uso destas Bibliotecas Digitais, disponíveis em https://digitalis.uc.pt/pt-pt/termos.

Conforme exposto nos referidos Termos e Condições de Uso, o descarregamento de títulos de acesso restrito requer uma licença válida de autorização devendo o utilizador aceder ao(s) documento(s) a partir de um endereço de IP da instituição detentora da supramencionada licença.

Ao utilizador é apenas permitido o descarregamento para uso pessoal, pelo que o emprego do(s) título(s) descarregado(s) para outro fim, designadamente comercial, carece de autorização do respetivo autor ou editor da obra.

Na medida em que todas as obras da UC Digitalis se encontram protegidas pelo Código do Direito de Autor e Direitos Conexos e demais legislação aplicável, toda a cópia, parcial ou total, deste documento, nos casos em que é legalmente admitida, deverá conter ou fazer-se acompanhar por este aviso.

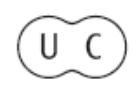




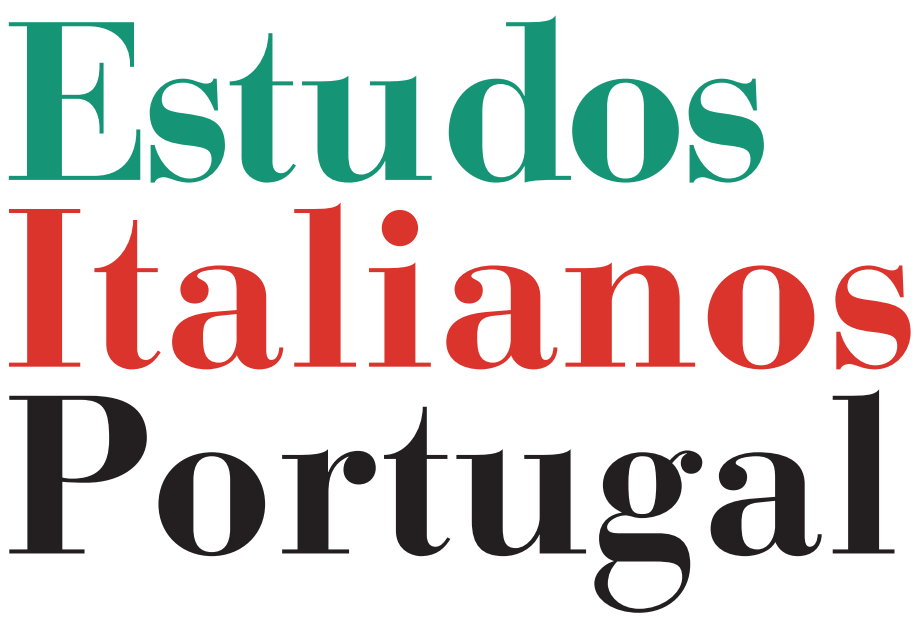

Instituto

Italiano

de Cultura

de Lisboa

Nova Série

$\mathrm{N}^{\mathbf{0}} 5$ 
La lingua italiana in Portogallo riscuote già da tempo enormi consensi che si sono tradotti, nell'ultimo decennio, in una significativa crescita del numero degli iscritti ai Corsi di Lingua e Cultura Italiana, non solo presso l'Istituto Italiano di Cultura ma anche presso gli Atenei locali. Infatti, nel corso degli anni l'italiano si è affermato sempre di più sia come lingua di cultura sia come lingua funzionale al mondo degli affari e del turismo in stretto rapporto con il processo di espansione in terra lusitana della nostra economia e dell'immagine del nostro Paese.

A conferma di questa tendenza positiva e dell'interesse manifestato in loco per la nostra lingua, si riportano i dati dei Corsi di Lingua e Cultura Italiana dell'Istituto di Cultura che per l'anno 2010 risultano 57, impartiti da 11 docenti e che vedono un pubblico discente che oscilla tra livelli e tipologie che vanno dall'età scolare a quella più avanzata, raggiungendo la cifra di 400 alunni per quadrimestre. I Corsi di Lingua quadrimestrali e intensivi, impartiti presso l'Istituto Italiano di Cultura, sono adeguati ai livelli stabiliti dal

* L'Istituto Italiano di Cultura di Lisbona, organismo ufficiale dello Stato italiano, ha quale obiettivo quello di promuovere e di diffondere la lingua e la cultura italiana in Portogallo attraverso l'offerta di Corsi di lingua e l'organizzazione di eventi culturali, anche in collaborazione con le istituzioni locali, al fine di favorire la circolazione delle idee, delle arti e delle scienze. 
Quadro Comune Europeo di Riferimento per le Lingue del Consiglio d'Europa (Common European Framework) e sono articolati in sei livelli di competenza: A1, A2, B1, B2 (con 60 ore ciascuno) e C1 e C2 (con 120 ore ciascuno) per un totale di 480 ore. Oltre all'offerta di Corsi di Lingua, l'Istituto propone anche Corsi di Italiano Commerciale, Corsi di Conversazione, Traduzione, Laboratorio di Scrittura, Corsi per Bambini italo-portoghesi e Seminari Tematici di Storia dell'Arte, Storia dell'Opera, Storia del Cinema e Letteratura Italiana.

L'Istituto Italiano è altresì sede degli Esami di Certificazione CILS e CIC dell'Università per Stranieri di Perugia, e CELI dell'Università per Stranieri di Siena con due sessioni annuali. Da giugno di quest'anno, l'Istituto ha aderito alla rete del Consorzio ICoN, in collaborazione con l'Università di Pisa, offrendo l'opportunità di sostenere gli esami per via informatica presso la propria sede.

Dal punto di vista strettamente culturale, l'Istituto Italiano di Cultura di Lisbona consolida la sua prestigiosa tradizione nel tessuto culturale metropolitano attraverso la programmazione di una vasta gamma di manifestazioni, quali conferenze, presentazioni letterarie, cicli cinematografici, rappresentazioni teatrali, spettacoli di danza, concerti di musica classica e contemporanea, mostre d'arte, in alcuni casi in collaborazione con altri Centri culturali portoghesi. Tra il numeroso pubblico sempre presente si nota una forte adesione da parte degli alunni che frequentano l'Istituto, a testimonianza di una maggiore sensibilizzazione agli eventi culturali che ha permesso di superare la dicotomia che vedeva un tempo l'attività dei Corsi separata dalle manifestazioni.

Per ciò che concerne lo stato della lingua italiana a livello accademico, l'insegnamento della nostra lingua, oltre che presso i tre Lettorati di ruolo dell'Università di Lisbona, Nova di Lisbona e di Évora, si tiene a livello curricolare e opzionale presso l'Università dell'Algarve in Faro, con Corsi 
di Lingua attivati grazie ad un contributo quinquennale del Ministero degli Affari Esteri, concesso a seguito della chiusura del Lettorato di ruolo. Corsi di Lingua italiana sono presenti anche presso la prestigiosa e antica Università di Coimbra, del Minho a Braga, delle Azzorre e di Aveiro, nonché presso le Università private Autonoma, Cattolica, Lusofona e della Terza Età.

Non sono presenti comunque Cattedre di Italiano presso le Università portoghesi, ad eccezione dell'Università di Coimbra, né esistono in loco scuole italiane o bilingui. Tuttavia, a livello di scuola secondaria, l'insegnamento della nostra Lingua è previsto nel piano di studi del locale Liceo Francese "Charles Lepierre" di Lisbona, dove dall'ottobre del 1985 viene impartito un Corso di Italiano per 15 ore settimanali per un numero complessivo di 60 alunni all'anno, dell'Istituto Superiore di Turismo di Estoril e delle Scuole di Musica del Conservatorio, e si segnala la presenza di Corsi di lingua italiana a livello sperimentale anche in altre istituzioni scolastiche.

Se si considera che la lingua italiana non è materia curriculare nel sistema scolastico portoghese a differenza di altre lingue come inglese, francese, tedesco e spagnolo, emerge lo sforzo richiesto ai centri di insegnamento per l'italiano che rispondono in maniera soddisfacente alla domanda di Corsi di Lingua e Cultura Italiana della popolazione portoghese. Al ruolo decisivo svolto dall'Istituto Italiano di Cultura di Lisbona ai fini della promozione e diffusione della lingua e cultura italiana è doveroso quindi affiancare l'impegno costante profuso dai Lettori ministeriali i quali, oltre a svolgere attività didattica, collaborano con l'Istituto alla promozione di attività culturali, assicurando in tal modo una più incisiva proiezione della lingua e della cultura in seno agli Atenei locali.

Nel corso del presente anno accademico, presso l'Università di Lisbona si sono registrati tre Corsi di livello base 
ed intermedio con complessivi 35 alunni, mentre all'Università Nova sono stati attivati cinque Corsi di livello base, intermedio ed avanzato per un totale di 83 alunni. Per quanto riguarda, invece, la realtà della prestigiosa Università di Évora, recentemente si è assistito ad un incremento considerevole di accordi internazionali attraverso gli scambi Erasmus e, di conseguenza, di numero di studenti di Lingua Italiana per un totale di 48 alunni divisi in 3 Corsi di livello base ed intermedio. Dopo l'Ateneo di Faro, anche presso quello di Évora non sarà più presente dal prossimo anno accademico il Lettore italiano di ruolo, a seguito degli interventi di riduzione di spesa realizzati dal Ministero degli Affari Esteri. Relativamente a Faro, l'Università si avvale da tre anni di un contributo ministeriale quinquennale e della presenza di un'insegnante di madre lingua contrattata direttamente: anche in questo caso l'insegnamento della Lingua e Cultura italiana rientra nelle scelte di una seconda o terza lingua non essendo presente un Dipartimento di Italiano. Le attività didattiche della lettrice e la vivacità organizzativa del Dipartimento di Lingue Moderne hanno incrementato il numero degli alunni interessati alla nostra cultura: 50 alunni per tre Corsi di lingua di livello base ed intermedio. Il contributo ministeriale assegnato rappresenta un tentativo di incentivare la crescita del numero di studenti di Lingua e Cultura italiana in una realtà decentrata, ma strategica dal punto di vista turistico-economico.

Diversa distribuzione della presenza dell'insegnamento della lingua italiana si può osservare nelle realtà più a Nord del Portogallo. Nella città di Coimbra esiste da tempo un prestigioso Istituto di Studi Italiani che garantisce non solo un Corso di Laurea in Lingua e Letteratura italiana, ma rappresenta un fondamentale punto di aggregazione scientifica per la vivacità dei componenti del Dipartimento, guidati dalla Professoressa Rita Marnoto. Nella città di Oporto, che ha avuto un grande rilancio dopo essere stata proclamata 
nel 2001 Capitale della Cultura Europea, divenendo sede di importanti centri culturali, dopo la chiusura della sezione distaccata dell'Istituto Italiano di Cultura nel 1993, non risulta esserci una presenza significativa dell'insegnamento della nostra lingua sia a livello superiore che universitario, con un'evidente debolezza ed inammissibile "vacanza" rispetto alle altre lingue europee. È pur vero che la presenza di un Consolato Onorario particolarmente attento e propositivo cerca di rimediare a questa mancanza offrendo Corsi di lingua, gestiti dalla Camera di Commercio, peraltro non sufficienti a coprire l'accresciuta domanda che si registra già da tempo. Infine, per quanto attiene all'Università del Minho, con sede nella città di Braga, nell'anno in corso si sono registrate interessanti iniziative da parte dei docenti dell'Area di Italiano volte a sensibilizzare gli studenti e ad avvicinarli alla nostra lingua e cultura.

Sebbene in terra lusitana la diffusione della lingua e della cultura italiana non appaia del tutto uniforme, evidenziandosi zone con una maggiore distribuzione di centri di insegnamento a scapito di altre dove se ne registra una presenza isolata e talvolta sporadica, si può concludere che, a conferma di un trend generale, anche in Portogallo, come in altri nazioni europee, si è passati da un pubblico genericamente interessato alla lingua italiana come investimento culturale ad un pubblico alla ricerca di un investimento formativo, dettato dalla maggiore spendibilità sociale della competenza in italiano come lingua straniera. 